\title{
Harmonic Interference of Earth's Orbital Velocity and the Sidereal Cosmic Ray Anisotropy
}

\author{
J.C. Díaz Vélez, ${ }^{a, b, *}$ P. Desiati, ${ }^{a}$ R. Abbasi ${ }^{c}$ and F. McNally ${ }^{d}$ \\ ${ }^{a}$ University of Wisconsin - Madison, U.S.A. \\ ${ }^{b}$ Universidad de Guadalajara, México \\ ${ }^{c}$ Loyola University Chicago, U.S.A. \\ ${ }^{d}$ Mercer University, U.S.A. \\ E-mail: juancarlos@icecube.wisc.edu, desiati@icecube.wisc.edu
}

\begin{abstract}
When cosmic-ray arrival directions are observed in celestial coordinates, they appear to have a small anisotropy whose origin is still largely unknown. In addition to this celestial anisotropy, the Earth's revolution around the Sun produces a faint Compton-Getting dipole anisotropy with an excess oriented towards the direction of motion in solar coordinates. The relative rotation of the celestial and solar reference frames over a calendar year causes interference between the two sources of anisotropy. It is possible to characterize the resulting yearly modulations by studying the side-bands to the diurnal and sidereal frequencies in anti- and extended-sidereal time frames. This work provides a numerical simulation of the interference between anisotropies in sidereal and solar reference frames to predict the distributions in anti-sidereal and extended-sidereal frames.
\end{abstract}

$37^{\text {th }}$ International Cosmic Ray Conference (ICRC 2021)

July 12 th - 23rd, 2021

Online - Berlin, Germany

\footnotetext{
*Presenter
} 


\section{Introduction}

Galactic cosmic ray particles arriving on Earth have been known to possess a small but measurable anisotropy in their arrival direction distribution (see [1] and references therein). Although observed already in a long time, it's in the last couple of decades that large ground-based experiments have provided detailed celestial coordinate sky maps of galactic cosmic rays from $\mathrm{TeV}$ to tens of PeV. In addition, recent observations of a large anisotropy at ultra-high energy provided the first hint of the transition from galactic to extra-galactic cosmic ray particles [2, 3].

Generally speaking, the observed cosmic-ray anisotropy (CRA) is due to a combination of extra-terrestrial and local effects. The extra-terrestrial contribution includes the distribution of the cosmic ray sources in the Milky Way, and the properties of the galactic and interstellar magnetic field, such as turbulence and the number and characteristics of coherent magnetic structures, including the heliosphere [4-6]. It is possible that the Compton-Getting effect due to the galactic rotation may be contributing to the observations [7], however, no evidence was found yet. Experiments' instabilities may produce locally generated modulations in the collected event rates . In addition, diurnal and seasonal variations of atmospheric pressure and temperature, which affects the production and propagation of secondary particles in the air showers initiated by cosmic ray particles hitting Earth's atmosphere, produce modulations in the collected event rates, as well. Finally, Earth's revolution around the Sun produces a spurious Compton-Getting dipole anisotropy oriented along the orbital motion's direction. All these locally generated modulations generate spurious anisotropy contributions that interfere with the sidereal CRA.

Since we are interested in unveiling the extra-terrestrial origin of the galactic CRA, it is necessary to understand and subtract all local contributions. A method to eliminate regularly modulated spurious local effects interfering with the sidereal anisotropy observations was developed by Farley and Storey [8] (see also [9]).

This work comprehensively addresses the effect that Earth's orbital motion has on the time modulation of the sidereal CRA, including the deformation actions of Earth's axis tilt and time variability of Earth's orbital velocity. A method to properly compensate for such a spurious distortion of the CRA is presented.

\section{Time modulations and frequency side-bands}

An amplitude modulation of a sinusoidal carrier wave with frequency $\omega_{f}$ can be interpreted as the superposition of the carrier wave and two waves with side-band frequencies $\omega_{f} \pm \omega_{s}$, where $\omega_{s}$ is the modulation frequency (see figure 1).

The observed CRA is characterized as a modulation of the arrival direction distribution in celestial (or sidereal) coordinates. Although the observed anisotropy has a complex angular structure, let's assume here that it is a dipole (representing a simple diurnal variation of the cosmic ray intensity in sidereal time frame). Let's also consider the case where an additional diurnal variation of the event rate is present in the solar time frame (e.g., induced by atmospheric seasonal modulations). Since Earth's rotation on its axis is accompanied by orbital motion, the duration of a sidereal day is about 4 minutes shorter than a solar day, as illustrated on the left panel of figure 2 . This means that a solar diurnal variation is characterized by a cycle frequency of $\omega_{f}^{\text {sol }}=1 /$ day and a sidereal 


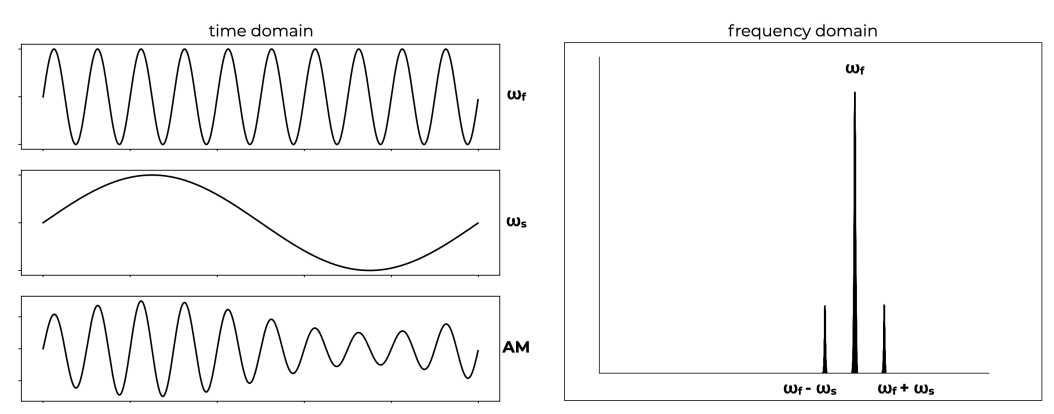

Figure 1: Left: a carrier wave with frequency $\omega_{f}$ modulated by a wave with frequency $\omega_{s}<\omega_{f}$ produces an amplitude modulated (AM) wave in time domain. Right: the AM wave decomposition in frequency domain, with the carrier frequency surrounded by side-bands with frequencies $\omega_{f} \pm \omega_{s}$.

diurnal variation by a cycle frequency of $\omega_{f}^{\text {sid }}=1.0027 /$ day ${ }^{1}$. The relative difference between the length of solar and sidereal days causes interference between the two sources of anisotropy over the course of a full orbital period. Such an interference is observed as an annual modulation (i.e. at frequency $\omega_{s}=(1 / 365.24$ days $)=0.0027 /$ day $)$ of the diurnal sidereal variation of the galactic cosmic ray intensity. As mentioned above, this modulation results in two symmetric side-bands at a frequency distance of $\omega_{s}$ around $\omega_{f}^{\text {sid }}$. Equivalently, the same interference can be observed as an annual modulation of the diurnal solar variation, thus producing side-bands around $\omega_{f}^{\text {sol }}$, as illustrated on the right panel of figure 2 .

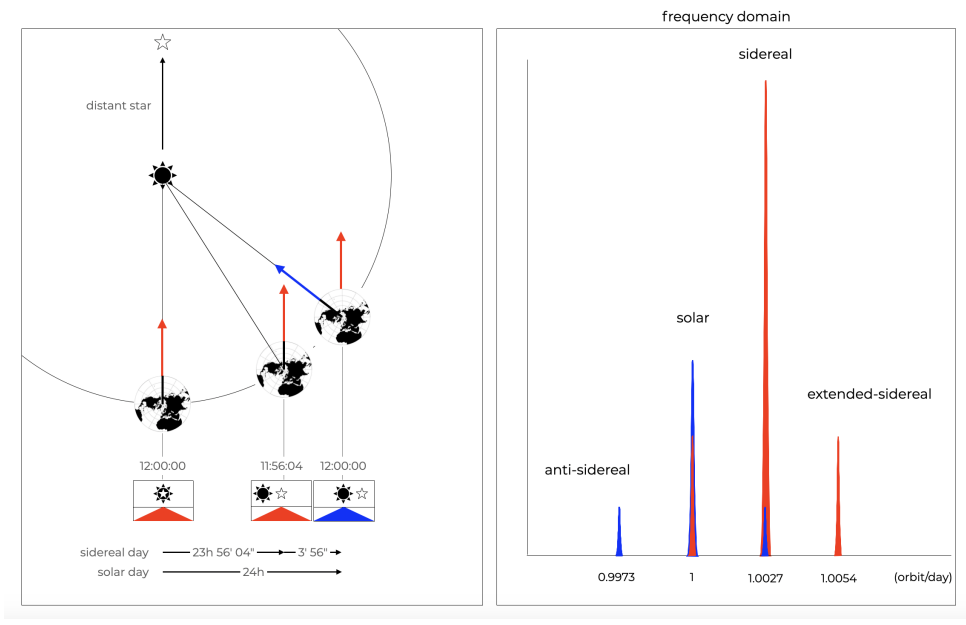

Figure 2: Left: the sidereal time with respect to solar time. Time starts at 12:00:00 on the local meridian with the Sun at the same location as a distant star. Then, as the Earth revolves around the Sun, the sidereal time zero point stays fixed in the celestial sky while the solar time's reference point moves away. They will not coincide at 12:00 again for one solar year. Right: the mutual interference between modulations in the solar and sidereal frames produces frequency side-bands around both peaks. The anti-sidereal and extended-sidereal side-bands do not overlap with any expected modulation frequency. On the contrary, the other side-bands overlap with the sidereal and solar frequencies, respectively, producing a deformation of the CRA.

\footnotetext{
${ }^{1}$ as a consequence, there are 366.24 sidereal days over the course of a complete orbit around the Sun.
} 
Figure 2 shows how the side-bands from the annual modulations of the interfering solar and sidereal variations overlap with $\omega_{f}^{\text {sid }}$ and $\omega_{f}^{\text {sol }}$. Only the so-called anti-sidereal and extendedsidereal side-bands exist where no extra-terrestrial originated modulation is expected. Therefore, these distributions are typically inspected to measure the extent of the interference modulation. Using methods such as Farley and Storey's [8], it is then possible to correct both the solar and sidereal modulation from the overlapping side-bands.

\section{The effect of Earth's orbital motion}

Let's assume that an experiment is stable over time, or that its variability is well known and can be taken care of. Let's also make the assumption that all atmospheric modulations are well understood and accounted for. Even so, the fact that Earth is moving on its orbit around the Sun at about $30 \mathrm{~km} / \mathrm{s}$ through the nearly isotropic rain of galactic cosmic rays, produces a relative excess rate in the direction of Earth's orbital motion with amplitude

$$
\frac{\Delta I}{I}=(\gamma+2) \frac{v}{c} \cos \theta
$$

with $I$ the cosmic ray intensity, $\gamma$ the cosmic ray spectral index, $v / c$ the ratio of Earth's orbital velocity to the speed of light, and $\theta$ the angle between the cosmic ray particle's arrival direction and the direction of motion [10].
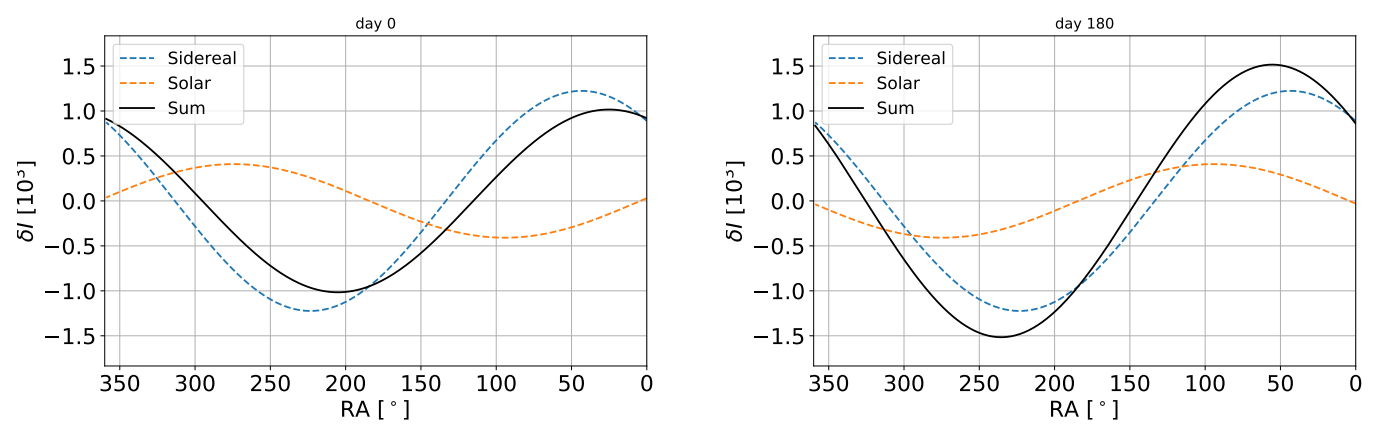

Figure 3: Interference between solar and sidereal dipole anisotropies as observed in the sidereal reference frame at two different times during the year. The solar dipole introduces a bias in both amplitude and phase of the sidereal dipole.

The relative rotation of Earth's orbital velocity with respect to the celestial coordinates produces an interference between the solar Compton-Getting dipole (referred to as solar dipole in the rest of the paper) and the sidereal anisotropy modulation. As explained in section 2, such interference causes a yearly modulation of the sidereal anisotropy due to the solar dipole rotation, as shown in figure 3, which is associated to the generation of frequency side-bands.

Assuming a uniform data collection livetime, this annual modulation should cancel after one full orbital cycle. The existence of such a modulation is however signaled by the presence of the anti-sidereal and extended-sidereal side-bands (see section 2).

The solar dipole, however, is not constant over the course of an orbital cycle, but it is affected by distortion modulations itself. One such modulation is the result of the tilt in Earth's rotation 


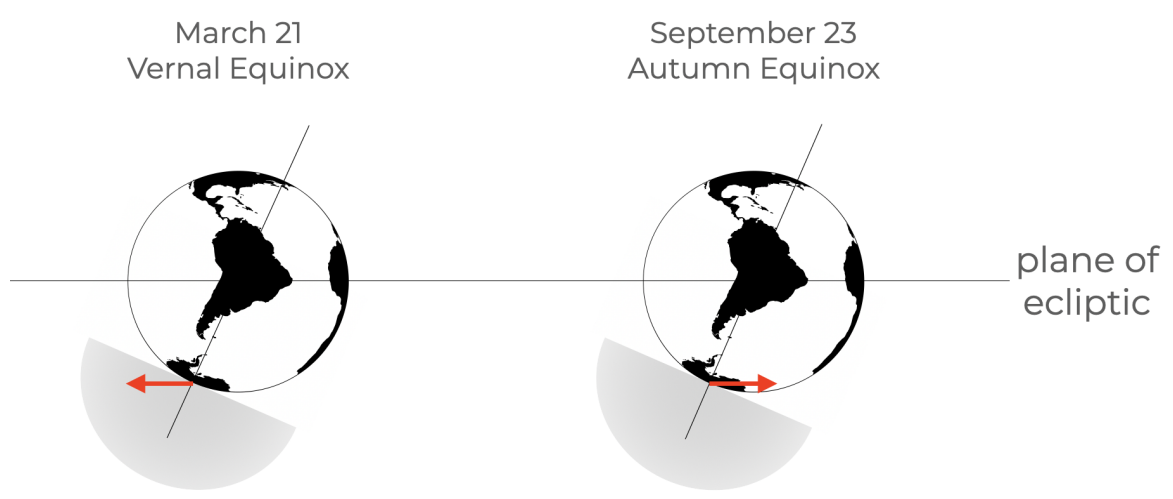

Figure 4: The Earth's orbital velocity vector (in red) swings between equatorial declinations of $\pm 23.5^{\circ}$ due to the planet's tilt with respect to the plane of the ecliptic. For experiments such as IceCube, located at the geographic South Pole, the orbital velocity vector swings between directions below and above the horizon.

axis with respect to the ecliptic plane, as illustrated in figure 4 . This results in a modulation of the solar dipole amplitude as observed at a given latitude. The amplitude has maxima at the winter and summer soltices, where the orbital velocity vector is aligned with the equator, and minima during the vernal and autumnal equinoxes, when the orbital velocity vector (or anti-vector) is below the horizon as can be seen in figure 5 .

In addition to this asymmetry, the magnitude of Earth's orbital velocity $v$ in equation 1 has a time dependence, primarily due to the eccentricity of Earth's orbit (and to a lesser extent, from the orbit around the Earth-Moon center of mass) as can be seen in the second panel of figure 5 .

\section{Conclusions}

There is a yearly modulation in the intensity of the solar dipole due to the eccentricity of Earth's orbit. As a result of this asymmetry, the interference of the sidereal and solar dipoles does not completely cancel out in one full orbital cycle and results in a small but non-negligible signal in the non-physical anti-sidereal and extended-sidereal frames. This variability has two separate components; one due to variable orbital speed $v$ in equation 1 and, in the case of a detector located at one of the poles with limited field of view (e.g. IceCube), there is a truncated dipole due to the obscuring of the maximum (minimum) below the horizon as a result of the current $23.5^{\circ}$ tilt in Earth's rotation axis relative to the ecliptic. The rate at which Earth's orbital velocity vector falls (rises) below (above) the horizon is not equal during the winter and summer solstices and this results in an asymmetric variation in amplitude. A simple toy simulation like the one shown in figure 6 reveals both the expected residual anti- and extended-sidereal distributions after integrating over 365 days.

The anti-sidereal and extended-sidereal distributions are commonly used as a systematic check on the stability of the detector throughout the year in CRA measurements. However, as we have demonstrated, even with a completely stable detector with no dead time or variability in acceptance, we can expect a dipole with amplitude of order $10^{-5}$ in the extended-sidereal frame and a slightly smaller one in the anti-sidereal frame. 

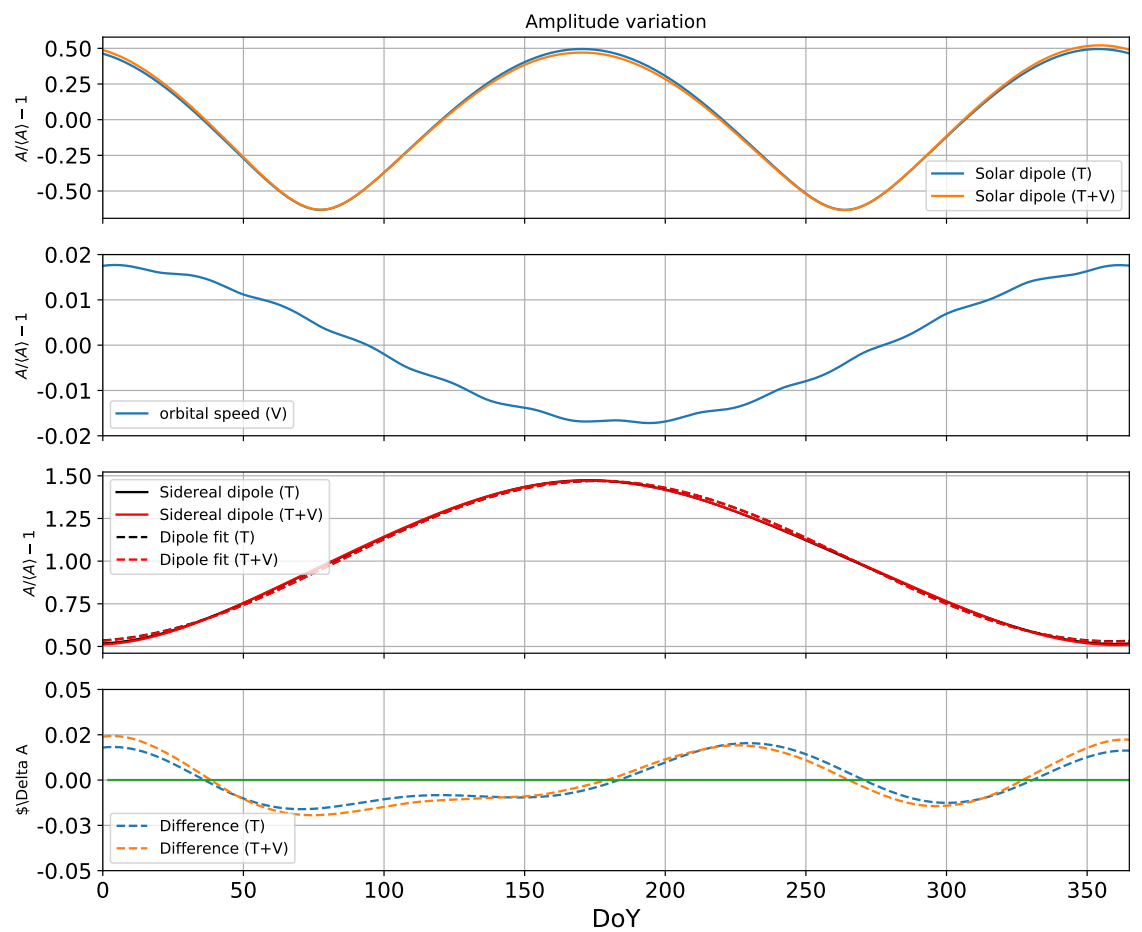

Figure 5: The tilt of Earth's rotation axis with respect to the ecliptic plane results in an asymmetry during the year such that the solar dipole doesn't quite cancel out after 365 days. An additional asymmetry in the amplitude of the solar dipole results the time dependence of the orbital velocity, primarily due to the eccentricity of Earth's orbit and to a lesser extent, from the orbit around the Earth-Moon center of mass. Top panel shows the Solar dipole amplitude variation as a function of time due to the tilt $(\mathrm{T})$ and including the effect of the orbital velocity $(\mathrm{T}+\mathrm{V})$. The second panel show the magnitude of the orbital velocity as a function of time. The third panel shows the amplitude variation of the sidereal dipole that results from interference with the solar dipole in both. A dipole fit results in a residual signal due to these asymmetries. The forth panel shows the difference between the dipole fit and the amplitude modulation of the sidereal dipole.

Both of these distributions are smaller or comparable to the statistical uncertainty of most published measurements but with enough statistics a careful measurement should be able to measure these distributions.

\section{Discussion}

As we have shown, we expect both an anti-sidereal and extended-sidereal distributions to remain present even with a completely stable detector with no dead time or variability in acceptance. This implies a biased measurement of amplitude and phase of both, the sidereal, and solar dipole distributions in CRA studies. One way to correct this, given that the intensity of the solar dipole is 

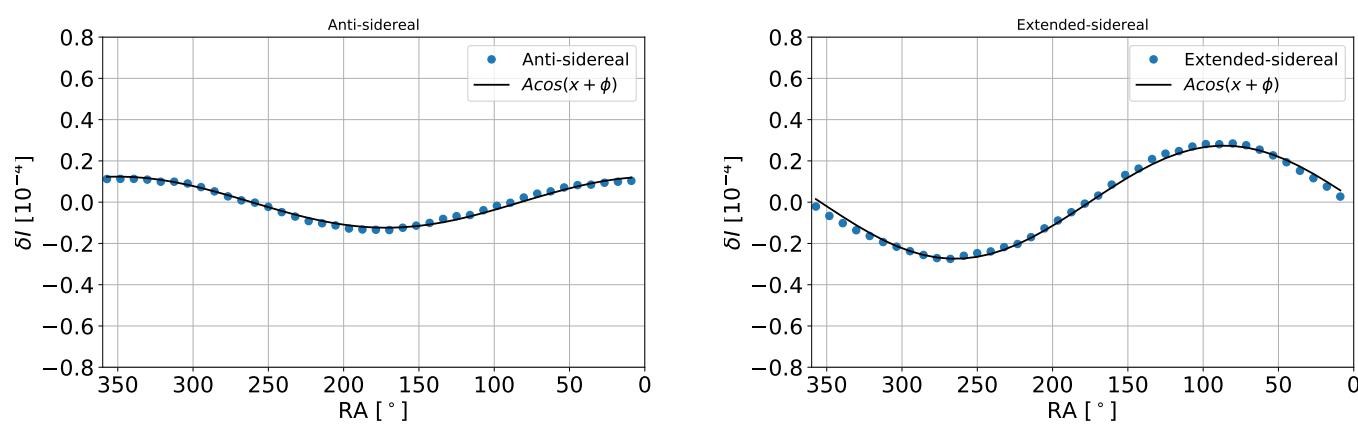

Figure 6: A toy simulation shows that, even with full coverage over 365 days, the solar and sidereal dipoles don't cancel out in their respective frames, resulting in a residual anti-sidereal (left), and extended-sidereal (right) distributions.

well understood and predicted by equation 1 by adding a compensation weight

$$
w_{i}=1-(\gamma+2) \frac{v\left(t_{i}\right)}{c} \cos \theta_{i},
$$

for each event $i$. This, however, does not consider possible biases in the reconstructed directions
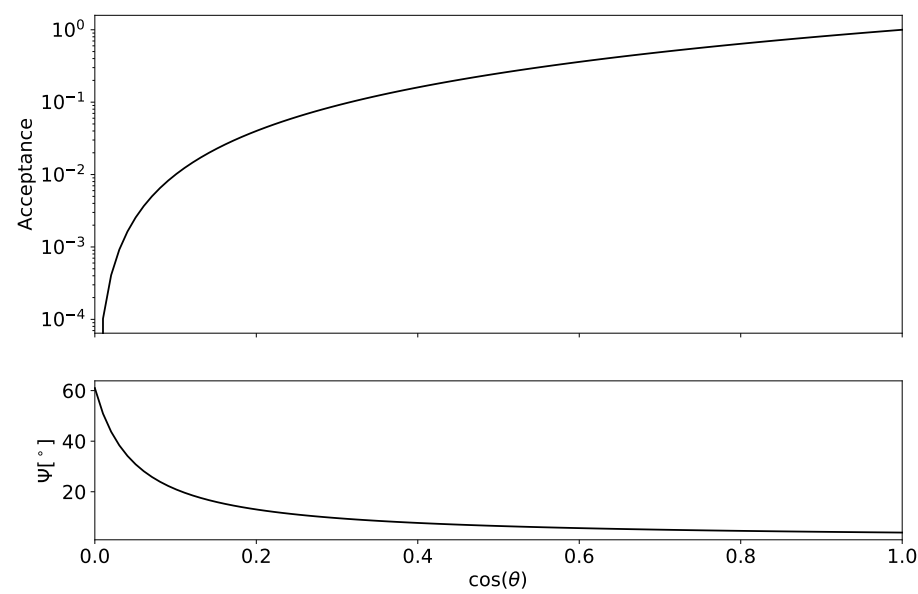

Figure 7: Top: Simple approximation to a typical detector acceptance as a function of zenith angle $\theta$ approximately follows a $\cos ^{2} \theta$ distribution [11]. Bottom: Toy model of angular resolution as a function of zenith angle $\theta$ similar to that described in [12].

of events. For typical Earth-based observations, the angular resolution tends to worsen towards the horizon as in the bottom panel of figure 7. This along with the variable acceptance as a function of zenith angle

$$
\frac{d N}{d \theta} \stackrel{\propto}{\sim} \cos ^{2} \theta,
$$

similar to the one shown in top panel of figure 7 results in a biased distribution towards larger zenith angles. As a result a simple application of the weight in equation 2 would result in an 

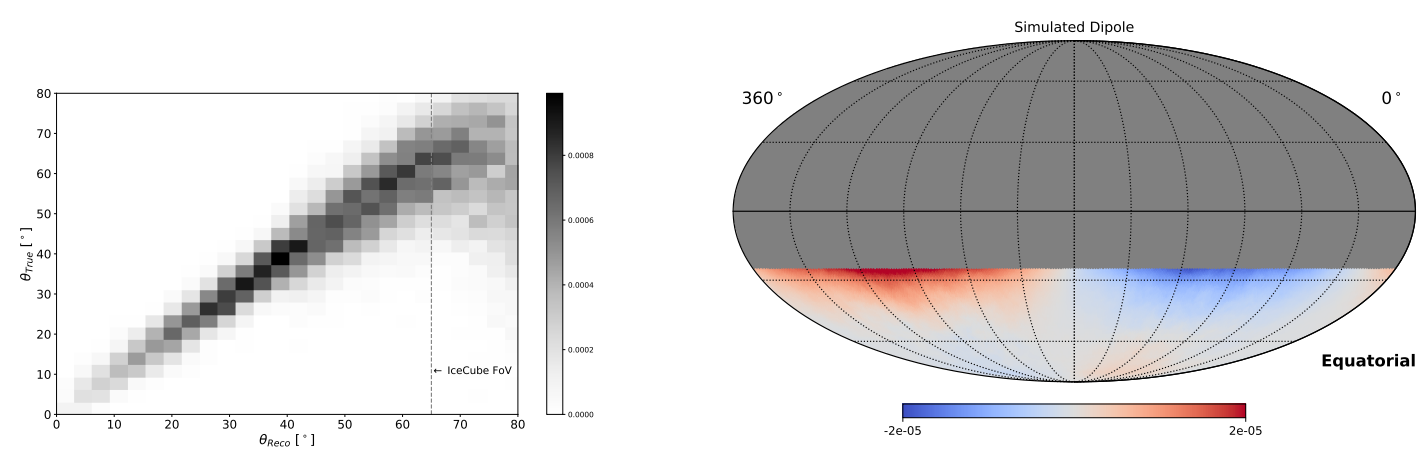

Figure 8: Left: Probability density function for a zenith angle $\theta_{T}$ to be reconstructed as zenith angle $\theta_{R}$. There is an increasing bias towards the horizon at $90^{\circ}$ that needs to factor into the solar dipole correction. Right: Zenith reconstruction bias results in an over-correction toward the horizon and an under-correction towards $45^{\circ}$.

over-correction for large zenith angles toward the horizon, and an under-correction towards $45^{\circ}$ where the acceptance per solid angle $d \Omega$ peaks. A correct compensation factor should include a correction based on the probability for a event to originate from a given direction as shown in figure 8. By eliminating biases in the measurement of amplitude and phase of sidereal, and solar dipole distributions, this approach has the added benefit of eliminating the requirement for full integer year coverage and may provide a tool for probing yearly variations in the sidereal cosmic-ray arrival distribution.

\section{References}

[1] McNally F., et al. (2021) ICRC2021, these proceedings

[2] Aab, A. et al., (2017), Science 357, 1266

[3] Abbasi, R.U. et al., (2018) Astrophys. J. Lett., 867, L27

[4] Díaz Vélez J.C. \& Desiati P. (2019) PoS(ICRC2019)1076

[5] López-Barquero V. \& Desiati P. (2019) PoS(ICRC2019)1109

[6] Desiati, P., et al., (2021), Snowmass 2021 Letter of Intent, arXiv:2009.04883

[7] Copton, A.H. \& Getting, I.A. (1935), Phys. Rev., 47, 817

[8] Farley F.J.M. \& Storey J.R., (1954), Proc. Phys. Soc. A 67, 996

[9] Guillian, G. et al., (2007), Phys. Rev. D 75, 062003

[10] Gleeson, L., \& Axford, W., (1968), Astrophysics and Space Science, 2, 431

[11] Shukla, P. \& Sankrith, S., (2018), arXiv:1606.06907

[12] Aartsen, M. G. et al., (2013), AAS, 765, 55 